\title{
An Audit of Occurrence of Dust in Tokamak and Stability of Fusion Plasma
}

\author{
Ayan Kumar MAKAR \\ Centre of Plasma Physics, Institute of Plasma Research, Sonapur, Assam 782402, India
}

(Received 17 October 2019 / Accepted 2 March 2020)

\begin{abstract}
Proliferation of dusty layers inside the Tokamak prompts deleterious effect in Tokamak operation. The stability of the fusion plasma is adversely affected by building-up of dusty layers inside the tokamak which actuate disruptions in tokamak's performance. The current study is based on probing the mechanisms of dust accumulation inside the Tokamak device by simulating the process with the support of pulsed plasma accelerator. Experimentally it was observed that upon interacting with the pulsed power plasma stream, the materials used for designing the in-vessel elements of Tokamak such as graphite, lost its crystalline structure which in turn affects its inherent properties like ductility, malleability, rust resistance etc., thus emanation of dust paricles take place as a result of collisions between plasma species such as electrons, ions and neutrals with molecules of graphite as the tokamak material, ergo causing erosion in the tokamak material. The concatenated dusty layers formed due to accumulation of dust particles inside the Tokamak can cause probable instability in fusion plasma that would mar the function of the Tokamak device, besides the generated dust would become chemically reactive, radioactive or toxic. The dynamics of dust grains can be realised by Coulomb force, ion drag force, frictional forces on ions, ion pre-sheath drifts etc. and can also be interpreted from the kinetic theory of dusty plasma, to account this the numerical studies have been carried out with DUSTT and UEDGE codes to understand the dust dynamics and transport mechanisms.
\end{abstract}

(C) 2020 The Japan Society of Plasma Science and Nuclear Fusion Research

Keywords: Tokamak, fusion plasma, dusty plasma, stability, DUSTT, UEDGE, radioactive, erosion

DOI: $10.1585 / \mathrm{pfr} .15 .1405019$

\section{Introduction}

The presence of dust particles in fusion devices is a growing interest since long time. However, their possible consequences for Tokamak operation and performances have become a topic of recent interest [1-3]. The paricles distinguished so far found to be between $10^{-9}$ to $10^{-4} \mathrm{~m}$ in size and presumed to be induced by erosion of walls, ion-molecular interactions, co-deposition arc, evaporation, sputtering and sublimation of wall material which are thermally overloaded [4-7]. Plasma impurities generally have two primary impacts on plasma efficiency: energy radiation and dilution of nucleus species, where the line radiation energy drop rises sharply with atomic number $(Z)$. The overall energy of radiation per unit time can be represented as $\Omega_{\mathrm{r}}=\mathrm{n}_{\mathrm{e}} \Sigma \mathrm{n}_{\mathrm{z}} \mathscr{R}\left(\mathrm{T}_{\mathrm{e}}\right)$, where $\mathrm{n}_{\mathrm{e}}$ and $\mathrm{n}_{\mathrm{z}}$ represents the number of electrons and ions respectively in the plasma and $\mathscr{R}$ is the parameter of radiation for the species under consideration. The energy dissipation due to Brake-radiation or bremsstrahlung is commensurate with the square of the atomic number of the species under consideration. Moreover, the dilution of fusion fuel is also due to plasma impurities. It was found that the fusion plasmas incline to be adulterated further at an increasing pace (coagulation of dusts) when contaminated. For instance,

author's e-mail: icerstark07@gmail.com the GEC informations have shown that rapid dust generation seems to boost plasma/wall conductivity, owing to sudden arcing and excessive dust extraction [8]. Advancing towards the surface, the sheath accelerates the impurity ions to a final impact energy given by $\varepsilon \propto z \phi_{\text {sheath }}+2 \mathrm{~T}_{\mathrm{i}}$, where the potential of the sheath $\phi_{\text {sheath }} \approx \frac{3.8}{e} \mathrm{~T}_{\mathrm{e}}, \mathrm{T}_{\mathrm{i}}$ and $\mathrm{T}_{\mathrm{e}}$ are the ionic and electronic temperature respectively of the plasma, $z$ is the charge of the ion and e is the charge of an electron. It is recognized that, inclusion to carbon atoms, the case of graphite wall components are important as well because of the release of crystal structures in the form of $\mathrm{C}_{1}, \mathrm{C}_{2}, \ldots, \mathrm{C}_{\mathrm{n}}$. Oxygen is particularly important in systems with graphite-covered surfaces among most impurities [9]. Chemically, oxygen sputtering shapes $\mathrm{CO}$ and $\mathrm{CO}_{2}$, even at room temperature, with a high output approximately to unity. The chemical sputtering is far stronger than physical sputtering in case of wall coated with graphite material, particularly at the graphite divertor boundary and in the target [10]. The interactivity of the plasma and the elements of the first wall and the divertor produces dust within the vacuum receptacle of a tokamak. In recent times, dust has received little publicity, primarily because it is neither a security issue nor an operating issue in current tokamaks. However, the construction of ITER emphasized on some of the basic demands for the next generation combustion plasma producing machine such as 
Table 1 Table with typical values of the pulsed power plasma and their system estimates.

\begin{tabular}{lll}
\hline S.No. & Quantities & Range \\
\hline $\mathbf{1 .}$ & Density of the Plasma & $\sim 10^{20}-10^{22} / \mathrm{m}^{3}$ \\
$\mathbf{2 .}$ & Velocity of the Plasma spurt & $\sim 18-20 \mathrm{~km} / \mathrm{S}$ \\
$\mathbf{3 .}$ & Density of the energy & $\sim 0.6-0.8 \mathrm{MJ} / \mathrm{m}^{2}$ \\
$\mathbf{4 .}$ & Discharge current & $\sim 100 \mathrm{kA}$ \\
$\mathbf{5 .}$ & Least diameter of the effective area & $\sim 0.04 \mathrm{~m}$ \\
6. & Anticipated density of power & $\sim 1.8 \mathrm{GW} / \mathrm{m}^{2}$ \\
\hline
\end{tabular}

understanding the occurrence of dust, Controlling the impurities, dust and related material mitigation, etc. to ensure appropriate quality of plasma facing components with enhanced plasma tolerance properties, besides dust is considered to be a possible safety hazard that is poisonous, radioactive and/or chemically reactive. The risk of contamination relies on the degree of dust generation and its confinement. Experimentally it has been found that substantial amount of hydrogen can be retained by tiny carbon fragment $(>0.2 \mathrm{H} / \mathrm{C})$, thus the dust can also affect the tritium inventories [2,3]. Furthermore the fine dust grains can also respond chemically and impulsively with oxygen and water vapours in the event of vacuum and/or coolant failure. Dust production and migration can probably serve a key function in core plasma contamination in and from the divertor region, for instance radioactive tritium capturing of dust grains and adverse impacts on tokamak's efficiency. The dust production level in a tokamak depends on the position and substance of the walls, they are composed of, thus dust production can be substantially decreased with the correct alignment and design of reference materials on the divertor and limiter. Accordingly this research is aimed at studying the dust production from the graphite material upon intereaction with highly energetic pulsed power plasma stream, their dynamics and transport mechanisms.

\section{Experimental Setup}

In this experiment the Pulsed Plasma Accelerator (PPA) can be categorized in five major parts Viz. pulsed power system, vacuum pumping system, vacuum chamber, electrode assembly and feeding system for gases. The pulsed power system consists of two capacitor bank modules, five capacitors in each module and ten capacitors in total, with each of $178 \mu \mathrm{F}$, were used to generate the high energy electrical pulse for time interval of $\sim 1 \mathrm{~ms}$, when discharging take place through ignitron, each of

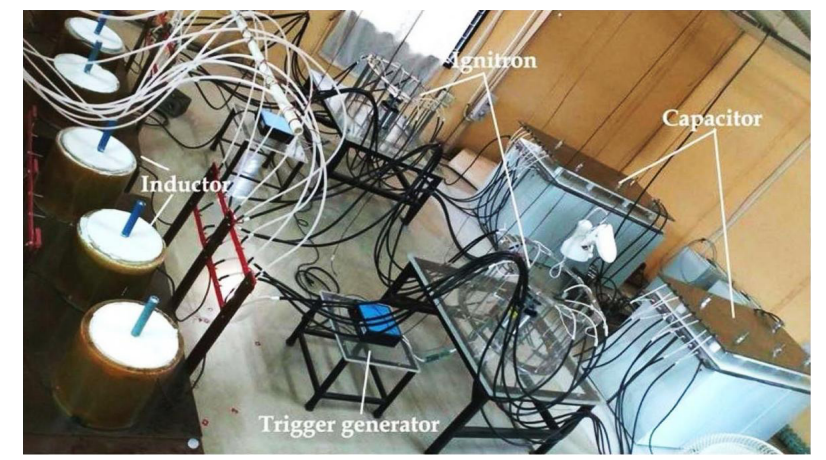

Fig. 1 Pulsed power system to operate pulsed plasma accelerator.

this module offers $50 \mathrm{KA}$ of current, the precise magnitude of current and time interval of discharging pulse is achieved by parallel combination of three inductance of $45 \mu \mathrm{H}$ each which can yield $\sim 15 \mu \mathrm{H}$ inductance overall (Fig. 1). Moreover parallel combination prevents the formation of high magnetic field with the passage of discharge current as it may damage the sensitive electrical elements. The ignitron act as a switch for both charging and discharging function of the capacitors. The High voltage pulse from high voltage power supply was fed into the capacitor bank through ignitron for charging purpose, when the bank is fully charged, the charging is discontinued and the ignitron is triggered by a high voltage to release the energy stored (about $200 \mathrm{~kJ}$ ) in the bank into the load, which is transmitted into the plasma producing chamber Via highly insulated coaxial cable. Plasma chamber have been divided into three parts, i.e. the electrode chamber in which the plasma is generated, the intermediate chamber in which the plasma is exposed to the magnet field and the target chamber in which properties of material is studied (Fig. 2). The pulsed plasma accelerator is a type of Coaxial Plasma 


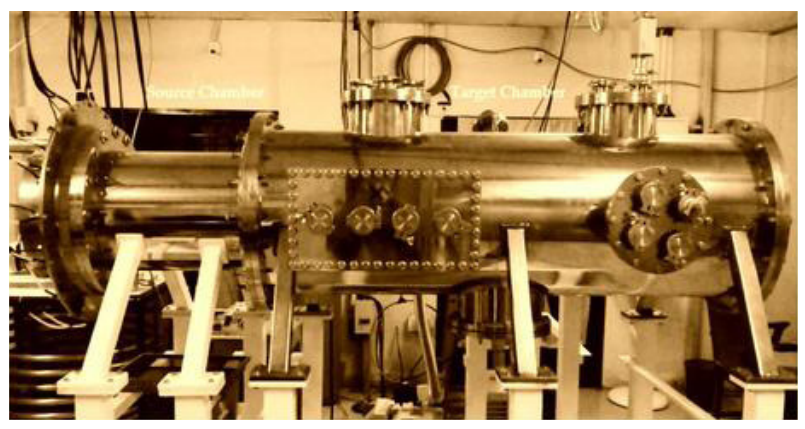

Fig. 2 The plasma Chamber - source chamber, intermediate chamber, Target chamber.

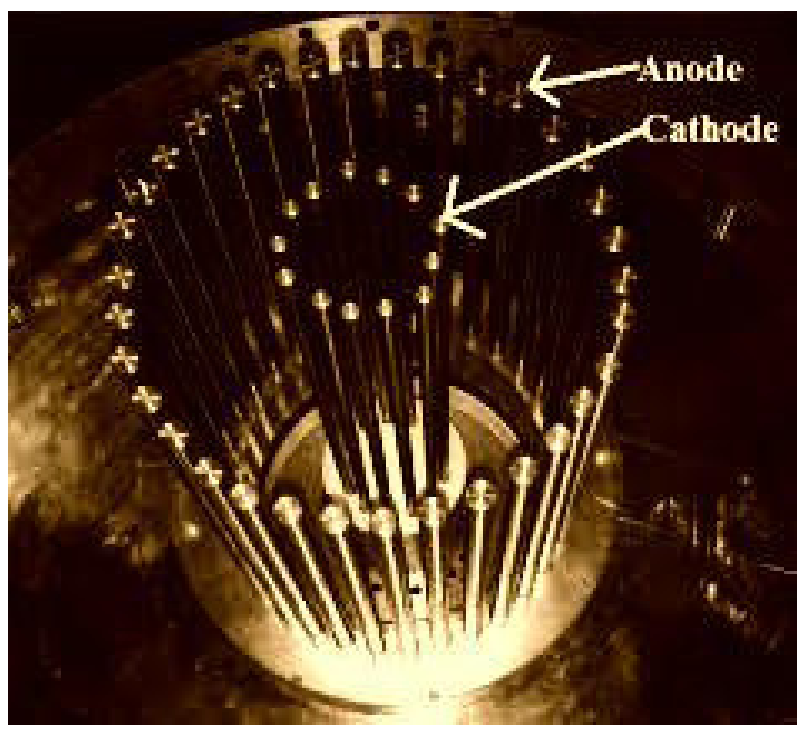

Fig. 3 The electrodes assembly, with centre cathode and outer anode.

accelerator system with two coaxial electrodes where the electrodes are sealed on one side and the other side is left free. One end of this chamber is attached to the target chamber and the other end is shut with an assembly of electrode. To achieve a uniform, diffused and high-density plasma distribution, the electrode assembly in the central region is made of cathode and the electrode assembly of the external region is made of anode (Fig. 3). In this coaxial electrode assembly there are 13 numbers of discrete SS electrode rods for cathode and 34 numbers of discrete SS electrode rods for anode, the radii of which are $0.025 \mathrm{~m}$ for cathode and $0.085 \mathrm{~m}$ for anode respectively. Based on this consideration the source chamber is constructed to be of length $0.0470 \mathrm{~m}$ with diameter $0.0360 \mathrm{~m}$ and target chamber of length $1.5 \mathrm{~m}$ with diameter $0.5 \mathrm{~m}$ respectively for effective propagation of plasma stream (Fig. 2). High vacuum is maintained inside the plasma producing chamber with the support of turbomolecular pump backed by rotary pump, and the pressure in the system was measured with the help of pirani and ionisation gauges. During the

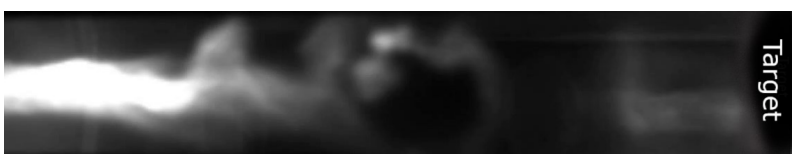

Fig. 4 The plasma-target interaction captured by high resolution camera iX Cameras - i-SPEED 716 with 500,000 fps max frame rate.

experiment $\sim 10^{-6}$ mbar of gas pressure was maintained inside the chamber. The gas is supplied with a gas injection valve that inserts the gas when the flyer plate of the plenum is opened where the gas is stored. When the voltage pulse is applied to the coil that attaches to the flyer plate, the gas flows within and fills the inter-electrode spacing. To attain the operating pressure a supersonic spurt of Argon gas is introduced with average mass flow rate of $\sim 50 \mathrm{gm} / \mathrm{s}, 4 \mathrm{~ms}$ before the discharge takes place. The current gas is electrically disrupted by the use of high-voltage pulses delivered from Pulsed Power system in between the electrodes that splits the gas into electrons and ions. This discharge produces a plasma sheath between the electrodes driven by auto-generated $\mathrm{J} \times \mathrm{B}$ force towards the open end. The plasma is generated between the inter-electrode spacing and driven to the target chamber towards the open end of the electrode by applying a negative $15 \mathrm{kV}$ high voltage pulse across the electrodes. The plasma then travels into the target chamber where a precise diagnosis is carried out for characterising the plasma, the plasma stream is then subjected on target samples for further analysis of material properties (Fig. 4). In this experiment the graphite is used as a target material, as up till now, graphite was most widely used as plasma facing material used for the lining of first wall or the divertor region of the Tokamak. The dust is formed as a result of energetic plasma/Target interactions. The existence of electromagnetic fields in the plasma greatly affected by the presence of dust grains and dramatically affects plasma characteristics. In addition to the electrons and ions found in ordinary plasmas, dusty plasmas comprise charged dust grains [11], as a result the turbulence is developed within a portion of the contaminated plasma leading to plasma instabilities, besides dust generated may pose significant safety issues, as it can be radioactive, chemically reactive or chemically toxic.

\section{Measurements of Energy Flux in Pulsed Power Plasma}

The time of flight method has been employed to calculate the energy flux of the pulsed power plasma as this technique found to be very useful for measuring the energy distribution of charged plasma particles. In this experiment a simple particle detector so called the retarding grid analyzer, of size $20 \mathrm{~cm}$ has been used. It consists of a series of electrodes which are separated by insulated washer from 
each other, the alloy used in fabricating this device is TZM (99\% molybdenum, $0.5 \%$ titanium, and $0.08 \%$ zirconium) because of its thermal stability and improved structural properties while the insulator used is hot-pressed Boron nitride as it possess both a large volume resistivity and an essentially zero coefficient of thermal expansion, the cables in retarding grid analyzer are made up of multiple layers of fiberglass and mica insulation which offers high resistance to heat and thus manifests thermal stability. The particle detector, is placed at a close proximity of the target material inside the plasma chamber, such that the time of arrival $(\tau)$ of the plasma particles can be recorded. The results then used to calculate the total energy (E) which is formulated as

$$
E=\frac{m}{2}\left(\frac{d}{\tau}\right)^{2},
$$

where $\mathrm{d}$ is the distance between the particle detector and the plasma source, $\mathrm{m}$ is the mass of plasma particles, $\tau$ is the time of flight.

The Retarding Grid Analyzer comprises a rolling disc to capture the charge plasma particles - electrons and/or ions, in good enough condition. A grid (an excellent porous metallic mesh) has been kept in front of a biased collector to prevent the particles of definite energies. The particles possessing energies below the biasing voltage are hindered and the remnants are captured by collector. When the biasing voltage is adjusted on the grid, the I-V curve is obtained, revealing the relationship between collector current (I) and biasing voltage (V). In the event of a changing positive grid potential, this device functions as an ion energy analyzer while it functions as an electron energy analyzer for variable negative bias. The plasma temperature can also be determined by the features of the I-V curve. The time development of the current $I(t)$ is formulated below

$$
I(t)=Q \cdot \frac{d N}{d T},
$$

$\mathrm{Q}$ is the charge of the particle, $\frac{d N}{d T}$ represents the time rate of change of number density of the particles collected in the disc.

The total number of particles collected in the disc as well as the corresponding total energy can be determined using (1) and (2) given below:

$$
\mathrm{N}_{\text {Total }}=\int_{0}^{\infty} \frac{I(t)}{Q} d T,
$$

also the expression of total energy is formulated as

$$
\mathrm{E}_{\text {Total }}=\int_{0}^{\infty} \frac{1}{2}\left(\frac{d}{\tau}\right)^{2} \cdot \mathrm{I}(\mathrm{t}) \mathrm{dt} \cdot \frac{m}{Q} .
$$

Essentially the spurious signal as a result of secondary emission from the grid must be reduced. Simple disk collector sometimes substituted with a Faraday cup to render narrow collection angle. Using this method the maximum energy of the pulsed power plasma stream found to be $100 \mathrm{~J} / \mathrm{cm}^{2}$, which corresponds to the energy to be received by the first wall of Tokamak during operation.

\section{The Mechanisms of Dust Forma- tion}

The dust produced due to plasma-Target interaction is of significant amount, with most particles of size $\sim 1 \mu \mathrm{m}$ have been found. The dust formation may be attributed to two major methods -1 . Bottom-up path, 2. Top-down path. Dust is produced by the bottom-up condensation method when atoms, molecules or tiny dust flakes $(\sim \mathrm{nm}$ range), coagulate and enhanced the growth of dust particulates, while it may also be produced when comparatively large parts of material break down into smaller segments by the Top-down method in single or multiple steps. In this experiment the target material (graphite) disintegrates into smaller fragments upon interacting with highly energetic pulsed plasma spurt which give rise to concatenation of dust layers inside the plasma target chamber. The dust thus formed are stored inside a container. A piezoelectric shaker has been used to introduce the dust grains into the plasma chamber from the container during the analysis of

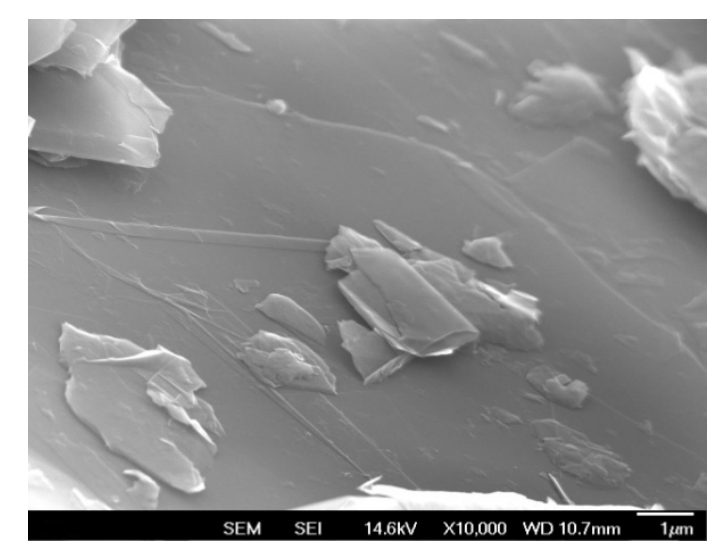

i.

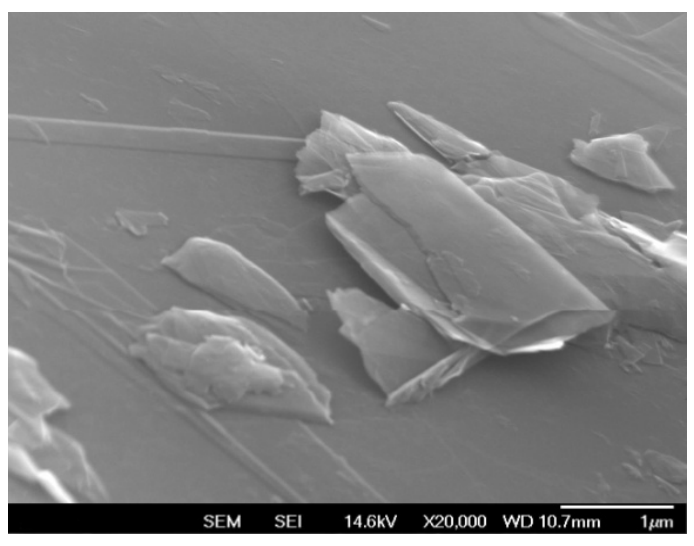

ii.

Fig. 5 Scanning Electron Microscopy (S.E.M) image of dust particle 1 , with size $\sim 1 \mu \mathrm{m}$, with magnification X 10,000 and of dust particle 2 , with size $\sim 1 \mu \mathrm{m}$, with magnification X 20,000. 
plasma-dust interaction. The distribution of tokamak dust in particle size and quantity has been assessed with SEM microscope images (Fig. 5). It has been found that most of the generated dust particles are of $1 \mu \mathrm{m}$ in size.

\section{Numerical Study of Dust Dynamics and Transport in Fusion Devices}

This experiment provides a simulation of dust formation and dust dynamics similar to the Tokamak device. The computerised codes used for analyse the dust dynamics and transport statistics are DUSTT and UEDGE. DUSTT code has been used to monitor the motion of dust flakes in three-dimensional (3D) geometry, by accounting parameters such as dust charge, momentum and energy balance of dust grains and its ablation. DUSTT utilizes entry information of plasma parameters such as heat flux, velocity, magnetic configuration etc. of the plasma species - electrons, ions, neutrals and impurity, straight from the 2D edge plasma transport code UEDGE, in which plasma parameters are pre-defined on $2 \mathrm{D}$ grids, for constructing the profiles of plasma parameters. The UEDGE code simulate the transport mechanism by employing multi-fluid approach. Therefore the simulation of the $3 \mathrm{D}$ transportation of dust grains in a genuine fusion plasma medium is probable with these codes.

When the collisions take place between the existing dust grains and plasma species, a force acts on the existing dust particles, which depends upon plasma flux, sizes of the dust grains, charges on dust grains, etc. This force arises due to combination of several forces - the gravitational force, the electromagnetic force, the drag force, the thermophoretic force and the radiation pressure force. The Monte-Carlo method is used to simulate the collision of dust grains and taking the ensemble average of the dust grains under consideration. In modelling, it has been assumed that the shape of dust particle is spherical and comprised of even solid substance with atomic density $\zeta_{d}$, total mass $\mu_{\mathrm{d}}$, total volume $\Gamma_{\mathrm{d}}$ such that, $\mu_{\mathrm{d}}=\Gamma_{\mathrm{d}} \cdot \zeta_{\mathrm{d}}$, where $\Gamma_{\mathrm{d}}=\frac{4 \pi R_{d}^{3}}{3}$, and the initial parameters (radius $\mathrm{R}_{\mathrm{d} 0}$, Temperature $\mathrm{T}_{\mathrm{d} 0}$, velocity $v_{\mathrm{d} 0}$ and birth point or point of injection) are the entry parameters for calculating the trajectory of dust particle. In this modelling the radius $\left(\mathrm{R}_{\mathrm{d} 0}\right)$ of the dust grains is taken to be $1 \mu \mathrm{m}$, which is almost the average mass-weighted radius of the collected dust grains. The total cross-section of the dust grains is assumed to be $\sigma_{\mathrm{d}}=\pi R_{d}^{2}$ with total surface area $\Lambda_{\mathrm{d}}=4 . \sigma_{\mathrm{d}}=4 \pi R_{d}^{2}$. The DUSTT code solves the coupled differential equations that represent the dynamics of dust grains under consideration. The equation of motions are:

$$
\frac{d \mathbf{r}}{d t}=\boldsymbol{v}, \quad \mu_{\mathrm{d}}(t) \frac{d v}{d t}=\mathrm{F}_{\mathrm{d}}+\Theta_{\mathrm{d}, \text { wall }} \cdot \boldsymbol{v}+\Theta_{\mathrm{d}, \text { turb }} \cdot \boldsymbol{v} .
$$

The force acted on the dust grains due to interaction with the plasma stream is represented as $\mathrm{F}_{\mathrm{d}}\left(\mathrm{r}(\mathrm{t}), v_{\mathrm{d}}(\mathrm{t}), \mathrm{T}_{\mathrm{d}}(\mathrm{t}), \mathrm{R}_{\mathrm{d}}(\mathrm{t})\right)$. The $\Theta_{\mathrm{d} \text {,wall }}$ and $\Theta_{\mathrm{d} \text {,turb }}$ are the operators which represents the collision of dust particles with the layers of first wall of the tokamak and the microturbulence of the plasma respectively. This operator is represented as

$$
\Theta_{\mathrm{d}}=\int \ldots \int d r d v d v^{\prime} \sum_{\varepsilon=1}^{\varepsilon=\epsilon s t} \delta\left(r-r_{\epsilon}\right) \delta_{\vartheta}\left(\vartheta_{\epsilon}, \vartheta_{\epsilon}^{\prime}\right) .
$$

The index $\varepsilon$ passes over a number of sites of collision, $\epsilon^{\text {st }}$ represents the maximum amount of collision sites on the trajectory, $\mathbf{r}_{\epsilon}$ is the radius of the collisional sites, $\vartheta=$ $\left(\mathbf{V}_{\epsilon}, \mathrm{R}_{\mathbf{d} \epsilon}, \mathrm{T}_{\mathrm{d} \epsilon}\right)$ and $\vartheta^{\prime} \equiv\left(\vartheta_{\epsilon}^{\prime}, R_{d \epsilon}^{\prime}, T_{d \epsilon}^{\prime}\right)$ represents the pre and post collisional state of velocity, radius and temperature,

$$
\begin{aligned}
\delta_{v}\left(\vartheta, \vartheta^{\prime}\right)= & \delta\left(\vartheta-\vartheta_{\epsilon}\right) \delta\left(\vartheta^{\prime}-\vartheta_{\epsilon}^{\prime}\right) \delta\left(\mathrm{R}_{\mathrm{d}}-\mathrm{R}_{\mathrm{d} \epsilon}\right) \delta\left(\mathrm{R}_{\mathrm{d}}^{\prime}-\mathrm{R}_{\mathrm{d} \epsilon}^{\prime}\right) \\
& \delta\left(\mathrm{T}_{\mathrm{d}}-\mathrm{T}_{\mathrm{d} \epsilon}\right) \delta\left(\mathrm{T}_{\mathrm{d}}^{\prime}-\mathrm{T}_{\mathrm{d} \epsilon}^{\prime}\right) .
\end{aligned}
$$

The DUSTT code also solves the conditions for temporal evolution which obeys:

$$
\frac{d H_{d}}{d t}=\frac{d C_{d} \mu_{d} T_{d}}{d t}=4 \pi R_{d}^{2} q_{\text {total }}
$$

Here $H_{d}$ is the enthalpy, $C_{d} T_{d}$ is the heat capacity, $\mu_{d}$ is the mass, $\mathrm{R}_{\mathrm{d}}$ is the radius, $q_{\text {total }}$ is the total heat flux.

For non black body approximation,

$$
q_{\text {total }}=q_{\text {plasma }}-q_{\text {eject }}=q_{\text {in }}-q_{\text {out }} .
$$

Immediately after the dust-plasma interaction, the variance in mobilities of plasma species (electrons/ions) source some charges on the dust grains to acquire, and thus a sheath is developed around the charged dust species. To determine the mechanism of charging acquired by the dust grains we need to determine the floating potential which is the potential acquired by the charged dust grain when the current generated by both the electron and ion flux becomes equal (ambipolarity condition) and thus exactly cancel one another. The two methods of calculating the floating potential are the radial and orbital motion theory. The radial motion theory, also known as ABR theory is based on system having purely radial motion and involves no angular momentum. However for dust grains possessing low potential, angular momentum is of significant concern. Moreover the dust grains which are spherical in shape pose more complications in calculation of boundary conditions, as ABR theory correctly work only with planar geometry thus the solution of spherical system cannot be obtained in such cases. Considering the drawbacks of radial motion (ABR) theory, we use OML (orbital motion theory) to calculate the floating potential and to determine the charges on dust grains. In equilibrium condition of electron and ion flux at the surface, the surface potential $\Phi$ is calculated.

The total toroidal length $\mathrm{L}_{\mathrm{t}}=\int_{0}^{t}|\vartheta| d t$ and poloidal length $\mathrm{L}_{\mathrm{P}}=\int_{0}^{t}\left(\vartheta_{r}^{2}+\vartheta_{Z}^{2}\right)^{\frac{1}{2}} d t$ traveled by the dust particle reveals the trajectory of that dust particle and is represented in Fig. 6 versus time. The evolution of other parameters like temperature is represented in Fig. 7 and the normalised mass with particle trajectory $\left(\frac{L}{L_{P, M a x}}\right)$ is represented in Fig. 8. 


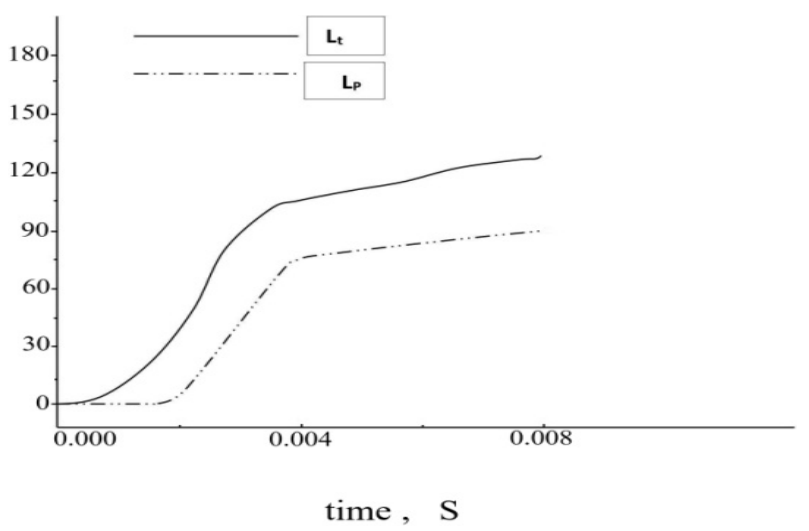

Fig. 6 The total travelled toroidal length VS time.

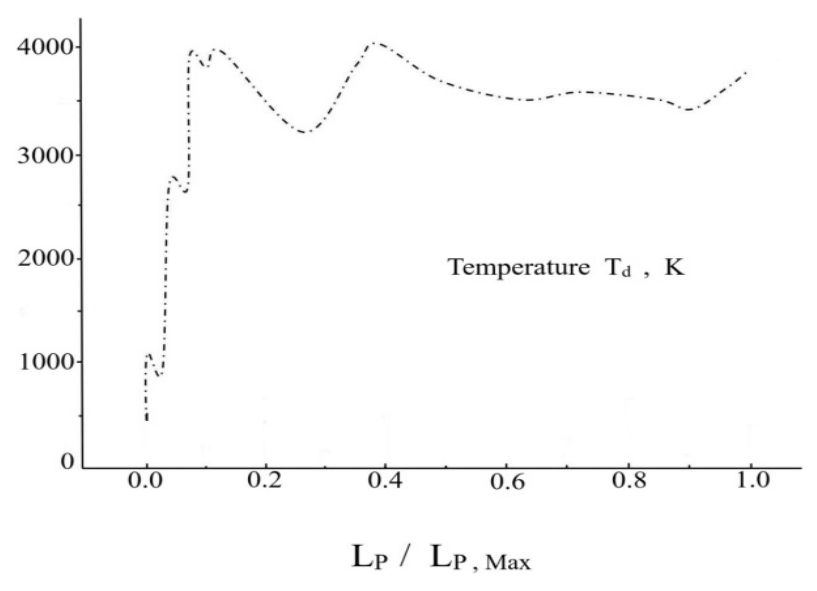

Fig. 7 The evolution of temperature with particle trajectory.

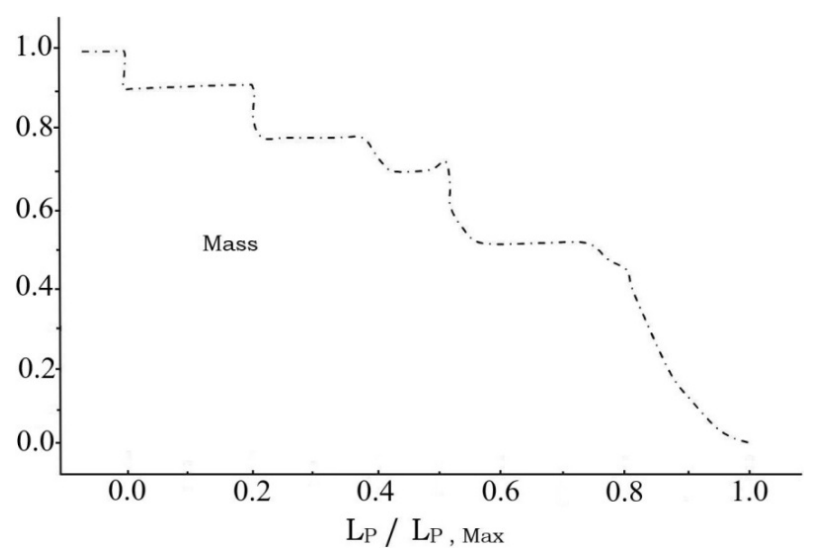

Fig. 8 The evolution of mass with particle trajectory.

The variation of heat flux in the dust particle along the particle trajectory is represented in Fig. 9 and the velocity of magnitude $|\vartheta|$ along the trajectory of dust particle is represented in Fig. 10. The variation of charge on a dust particle along with particle trajectory is represented in Fig. 11.

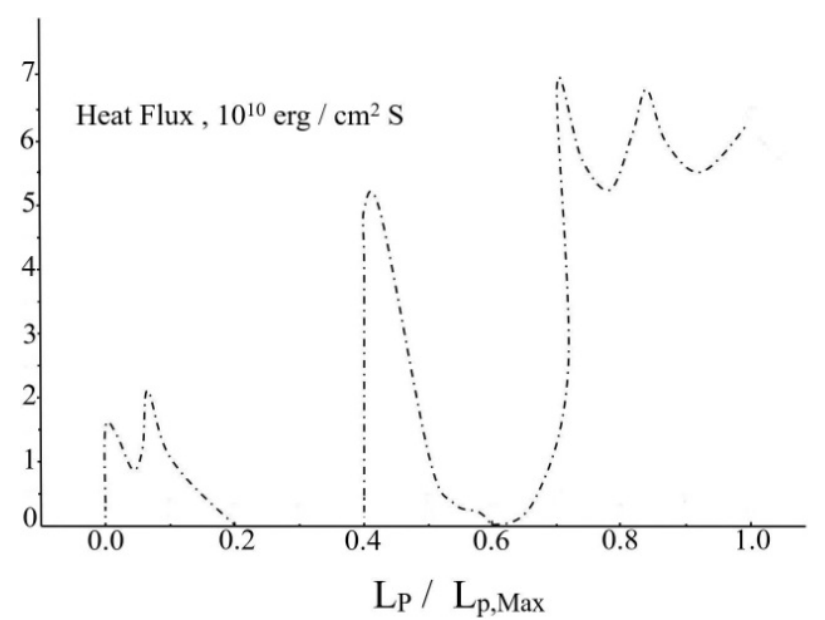

Fig. 9 The variation of heat flux with particle trajectory.

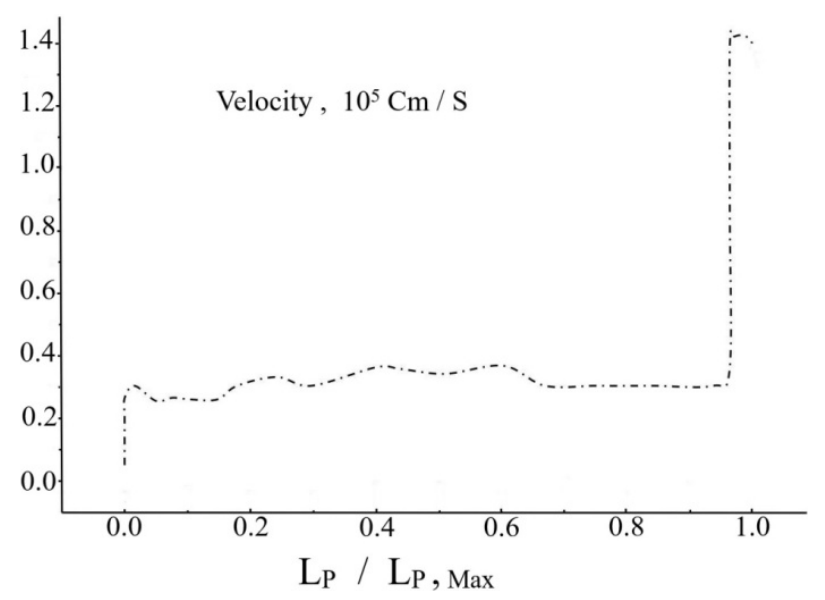

Fig. 10 The variation of velocity along the particle trajectory.

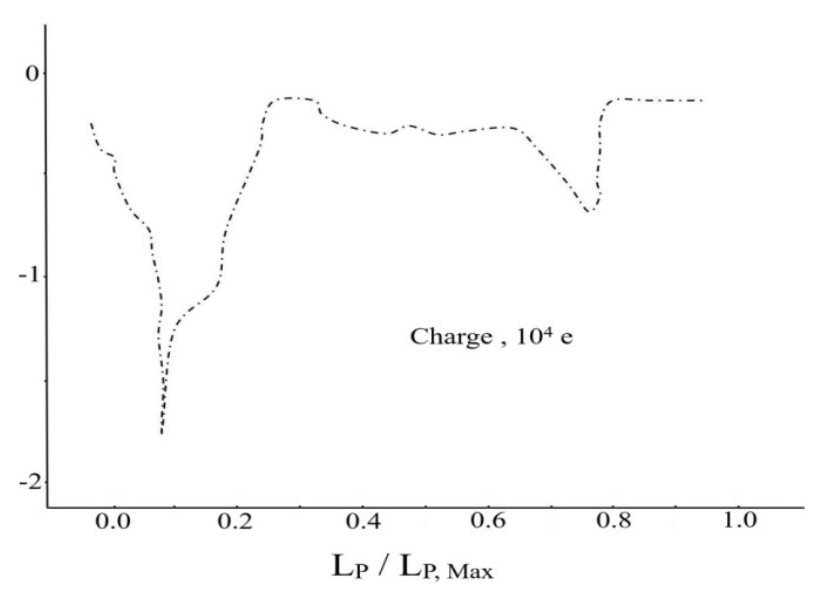

Fig. 11 The variation of charge along the particle trajectory.

\section{Results and Discussion}

In dust dynamics, the particle size performs an essential part. In this modelling the initial radius $\left(\mathrm{R}_{\mathrm{d} 0}\right)$ of the dust grains is taken to be $1 \mu \mathrm{m}$, which is almost the average 
mass-weighted radius of the collected dust grains. It has been found that the greater the radius, the more prolonged the lifetime of the dust particle with greater plasma penetrability (Fig. 6), thus, before evaporation, dust grains can reach quite deep in the hot plasma regions (Fig. 7). If the dust grains are expanded in size, the dust migrates further before it evaporates (Fig. 8). Consequently, a dust grain's overall retention time is the duration required to heat it to the transition temperatures besides the interval needed for the phase transitions of the dust grain. At a significantly lower temperature the graphite evaporates, yet it can persist for a consequential duration across the point of sublimation (Fig. 9). The velocity of plasma spurt can also alter the magnitude and orientation of dust partcles and even the faster dust grains can eventually penetrate the core plasma region (Fig. 10). A dust grain can therefore flee a heated plasma region and settle down into a cooler region. However, it can be expected that a substantial amounts of dust grains would have evaporated before this, which is why the dust accumulation is much lesser in some region and is not uniform throughout. The inspected trajectories illustrate the spread of the dust particles on one layer to other layers inside the Tokamak device as well. It can also be interpreted that the length of the radius $\left(R_{d}\right)$ of a spherical dust particle declines over time, that means the size of the dust paticle decreases over time as a result of the dust-plasma interaction while the charge on a dust particle increases with particle size and the plasma temperature (Fig. 11), here it has been assumed that the ionic temperature of the plasma $\left(\mathrm{T}_{\mathrm{i}}\right) \approx$ electronic temperature of the plasma $\left(\mathrm{T}_{\mathrm{e}}\right)$ as in case of fusion plasma. However as the fusion plasma density is high enough, the dust charging time in fusion plasma is usually very short, approximately, about $10^{-8} \mathrm{~S}$. Furthermore the dusty plasma comprises charged dust grains can develop turbulence in a specific region of the plasma leading to plasma instability, as the instability of fusion plasma largely rely on rate of dust contamination, these instabilities can couple to form $\mathrm{n}$ peeling-ballooning modes which in turn can form edge localised modes (ELM) thus affecting the magnetohydrodynamic (MHD) stabilities of the fusion plasma [12].

\section{Conclusions}

A simulation of dust formation inside the thermonuclear reactor has been carried out with the support of coaxial plasma accelerator. In this experiment the dust is found to be formed by plasma-target interaction, upon interaction, the target material (graphite) disintegrates into smaller fragments. The graphite is chosen as a suitable material for the target because of its enhanced thermomechanical characteristics and low atomic $(Z)$ numbers that makes the target material less vulnerable from contamination as compared to materials with higher atomic (Z) numbers [9]. The energy of the plasma spurt was determined using time of flight method, the maximum energy was found to be
$100 \mathrm{~J} / \mathrm{cm}^{2}$, which corresponds the energy to be received by the first wall of the Tokamak device during operation. Furthermore numerical studies have been carried out using DUSTT/UEDGE codes which reveals that the size of the dust particle performs an essential role in dust dynamics and transportation, it was found that the greater the size and velocity, the more prolonged the lifetime and plasma penetrability of the dust grains. Thus the faster dust grains can eventually migrate into the core plasma region before being evaporated. However the radius $\mathrm{R}_{\mathrm{d}}$ of a spherical dust particle declines over time as a result of the dust-plasma interaction. It can also be interpreted that the charge on a dust particle increases with particle size and plasma temperature. However as the fusion plasma density is high enough, the dust charging time in fusion plasma is very short, roughly about $10^{-8} \mathrm{~S}$. Dust production and migration can probably serve a key function in core plasma contamination in and from the divertor region, for instance radioactive tritium capturing in dust can adversely impact on device's efficiency, besides dust generated may pose significant safety issues, as it can be radioactive, chemically reactive or chemically toxic. This research thus presents a brief mechanism of dust formation and its dynamics concerning the stability of fusion plasma.

\section{Significance}

Dust produced in the thermonuclear reactor poses a major safety issue. consequently two major issues are associated with the fusion devices concerning dust generation, one of which concerns the operational safety of the fusion reactor; the other being accounted for plasma instability. The accumulation of dust grains induce concatenated dusty layers in the fusion device which can hinder the flow of heat energy into the cooling surfaces and also particularly affects the gaps constructed to facilitate electrical insulation or thermal expansion. Such layers get sublimate when subjected to immense heat loads [13]. On the other side, this can also have an adverse impact on plasma parameters and can enable the plasma contamination thus affecting the plasma stability [14]. Accordingly a logical question is put forward: what are the origins of dust particles and what are their mechanisms of generation inside the thermonuclear reactors? To response this it becomes absolute necessary of comprehending the discrete processes of overall dust formation from plasma-surface interactions. It is also a noteworthy aspect that significant units of plasma facing componenets (PFC) are coated with carbon based materials such as graphites or carbon composites due to their enhanced thermomechanical properties $[15,16]$. Therefore the present research reveals the dust production process in association with dust dynamics to estimate the stability of fusion plasma. 


\section{Acknowledgements}

The author is highly grateful to the centre director for providing the required facilities, project supervisor for his invaluable guidance and motivation and all the staffs for constant support to carry out this research. Finally, the author is deeply indebted to almighty and his parents for their blessings, his brother and sisters for their continuous support and sustained encouragements, all well wishers for their blessings and good wishes, which helped the author at every stage to complete this work.

[1] V.N. Tsytovich, Phys. Uspekhi 40, 53 (1997).

[2] J. Winter, Plasma Phys. Control. Fusion 40, 1201 (1998).

[3] J. Winter, Phys. Plasmas 7, 3862 (2000).

[4] S. Banerjee et al., Plasma Phys. Control. Fusion 60, 095001
(2018).

[5] G. Federici et al., J. Nucl. Mater. 283-287, 110 (2000).

[6] G. Federici et al., Nucl. Fusion 41, 1967 (2001).

[7] G. Federici et al., J. Nucl. Mater. 313-316, 11 (2003).

[8] J. Creel et al., "Particulate Contamination Within Fusion Devices and Complex (Dusty) Plasmas" IEEE 34th International Conference on Plasma Science (ICOPS) (2007).

[9] A. Miyahara and T. Tanabe, J. Nucl. Mater. 155-157, 49 (1988).

[10] N. Ohno et al., J. Nucl. Mater 390-391, 61 (2009).

[11] L. Spitzer, Astrophys. J. 93, 369 (1941).

[12] W.M. Tang et al., Nucl. Fusion 20, 1439 (1980).

[13] F. Andritsos et al., Fusion Eng. Des. 17, 409 (1991).

[14] M. Shoji et al., J. Plasma Fusion Res. 11, 2402056 (2016).

[15] P. Gasior et al., J. Nucl. Mater. 390-391, 585 (2009).

[16] S. Iwashita et al., Fusion Eng. Des. 88, 28 (2013). 